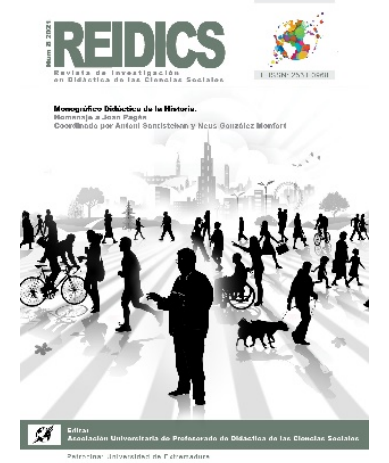

\title{
REIDICS
}

Revista de Investigación en

Núm. 8, 2021

Didáctica de las Ciencias

Sociales

Recibido 30 de febrero de 2021

Aceptado 31 de marzo 2021

E-ISSN: 2531-0968

\section{In memoriam del maestro Joan Pagès: Enseñar ciencias sociales para lograr un mundo mejor}

In memoriam of the master Joan Pagès: Teaching social sciences for a better world

Antoni Santisteban Fernández

Universitat Autònoma de Barcelona

Email: antoni.santisteban@uab.cat

ORCID: https://orcid.org/0000-0001-7978-5186

Jordi Castellví Mata

Universidad Internacional de la Rioja

Email: jordi.castellvimata@unir.net

ORCID: https://orcid.org/0000-0002-6487-5477

Neus González Monfort

Universitat Autònoma de Barcelona

Email:neus.gonzalez@uab.cat

ORCID: https://orcid.org/0000-0001-8597-0994

Gustavo González Valencia

Universitat Autònoma de Barcelona

Email: gustavo.gonzalez@uab.cat

ORCID: https://orcid.org/0000-0001-8718-417X

Mariona Massip Sabater

Universitat Autònoma de Barcelona

Email: mariona.massip@uab.cat

ORCID: https://orcid.org/0000-0002-7054-8819

DOI: https://doi.org/10.17398/2531-0968.08.8

\section{Resumen}

En esta introducción queremos repasar algunas de las temáticas esenciales que caracterizaron el trabajo de nuestro maestro Joan Pagès. A Joan el concepto de "maestro" le gustaba, consideraba que era una de las palabras más bonitas y de más alto rango que existen. De hecho, 
él fue, antes que nada, un maestro y así se definía con orgullo. Su gran preocupación fue siempre cómo mejorar la enseñanza y el aprendizaje de lo social, cómo educar una ciudadanía más crítica, más democrática, más comprometida. Así que haremos un pequeño recorrido por algunas temáticas sobre las que investigó y reflexionó, sobre las que debatió con entusiasmo $\mathrm{y}$ a las que hizo aportaciones que tanto nos han ayudado, a quienes creemos que la enseñanza de las ciencias sociales puede servir para lograr un mundo mejor. Recordamos aquí algunas de sus contribuciones más importantes, pero no pretendemos mostrar su obra, que abarca cerca de 400 publicaciones y una gran cantidad de temáticas, desde la enseñanza del tiempo histórico, la memoria y la conciencia histórica, el currículum, la formación del profesorado, la educación económica, jurídica, política, la educación para la ciudadanía, la participación, el trabajo a partir de problemas sociales o cuestiones socialmente vivas, la competencia social y ciudadana, el pensamiento social crítico, entre otras. Solo pretendemos señalar algunos aspectos de su trabajo, como ejemplo de la trascendencia de su obra. Esta introducción sirve de preámbulo a los distintos autores y autoras que han querido participar en este número de REIDICS, y que completan este monográfico de homenaje al maestro.

Palabras clave: enseñanza de las ciencias sociales; educación para la ciudadanía global; pensamiento social crítico; temporalidad; formación del profesorado.

\begin{abstract}
In this introduction we would like to review some of the essential themes that characterised the work of our master Joan Pagès. Joan liked the concept of "master", he considered it to be one of the most beautiful and highest-ranking words in existence. In fact, he was, first and foremost, a master and that is how he proudly defined himself. His great concern was always how to improve social teaching and learning, how to educate a more critical, more democratic, more committed citizenry. So we will take a brief look at some of the topics on which he researched and reflected, on which he debated with enthusiasm and to which he made contributions that have helped those of us who believe that the teaching of social sciences can serve to achieve a better world. We recall here some of his most important contributions, but we do not intend to show his work, which covers nearly 400 publications and a large number of topics, from the teaching of historical time, memory and historical awareness, curriculum, teacher training, economic, legal and political education, education for citizenship, participation, work based on social problems or socially live issues, social and civic competence, critical social thinking, among others. We only intend to point out some aspects of his work, as an example of the transcendence of his work. This introduction serves as a preamble to the different authors who have wished to participate in this issue of REIDICS, and who complete this monographic tribute to the master.
\end{abstract}

Keywords: social science teaching; global citizenship education; critical social thinking; temporality; teacher training.

Educar és una pràctica de l'hospitalitat

que té per missió acollir l'existència

(Garcés, 2020, p. 27)

\title{
1. Presencias e invisibilidades
}

Cantaba Amos Hart que un ser humano está hecho de algo más que aire, y que cualquier persona puede percatarse de la presencia de otra. Seguía lamentándose: "Mr. Cellophane should have been my name, cause you can look right through me, walk right by me, and never know I'm 
there". Con la canción evidenciaba una idea muy poderosa: no hay invisibles, la invisibilidad de personas y colectivos se genera en el desprecio de quienes miran.

Desde la línea de investigación de las presencias y las ausencias, de las invisibilidades o de las personas protagonistas e invisibilizadas de las ciencias sociales, se ha señalado el currículum de ciencias sociales como un órgano ideológico que reproduce valores nacionales, androcéntricos, eurocéntricos, coloniales y liberales (Massip, Castellví y Pagès, 2020). Contamos con un corpus extenso de investigaciones que constatan que los conocimientos sociales escolares se han articulado tradicionalmente a partir de los intereses de los estados-nación, que son considerados el objeto de las temáticas, y cuyos sujetos han sido sus élites dominantes. Un acto de poder genera exclusión e invisibilidad de la mayor parte de la humanidad, y supone un gran obstáculo para el reconocimiento y la dignificación de todas las personas, así como para la identificación del alumnado con aquello que aprende en las aulas de ciencias sociales.

Unos contenidos sociales estructurados sobre las dinámicas geopolíticas de los estadosnación europeos y sus minorías dirigentes conforman un mecanismo de reproducción de exclusiones y desigualdades. La limitación del protagonismo agéntico a las élites políticas y nacionales se ha asociado a la pedagogía histórica de la sumisión (Seixas, 2012). En este caso, la educación no cumple la tarea emancipadora y de denuncia y de lucha contra las desigualdades de las desigualdades, sino que las perpetúa. Santos (2019) defiende que la invisibilidad forma parte de las estrategias de dominación y de opresión de personas, colectivos y formas de vida. La entiende dentro la maquinaria de producción de la inexistencia para convertir "ciertos grupos de personas y formas de vida social" en "no existentes, invisibles, radicalmente inferiores o radicalmente peligrosos" (Santos, 2019, p. 53).

Revertir estas exclusiones e invisibilidades es un reto pendiente y urgente en la enseñanza de las ciencias sociales (Jara y Santisteban, 2018a). Según Villalón y Pagès (2013, p.121) "la enseñanza de la historia ha incorporado aspectos tan importantes como la conciencia histórica, la temporalidad, la memoria histórica, el patrimonio... sin embargo se ha dejado de lado el debate sobre los protagonistas". Aun así, este debate ha ido en aumento a lo largo de los últimos años, siendo en 2015 el tema central del Simposio de Investigación en Didáctica de las Ciencias Sociales de la AUPDCS ${ }^{1}$. Contamos ya con un corpus importante de investigaciones, artículos, tesis doctorales y propuestas para lograr una educación contra la exclusión y por el cambio social.

La investigación en este ámbito ha sido fundamental para comprender las dinámicas de exclusión en los currículos, los materiales educativos, las prácticas del profesorado o las representaciones del alumnado, entre otros, y también para analizar las posibilidades de cambio. Joan Pagès mostró una preocupación especial por las invisibilidades curriculares, y dedicó buena parte de su investigación, docencia y tutorización de tesis a la comprensión y a la erradicación de éstas, orientando la enseñanza de las ciencias sociales a la formación de la ciudadanía democrática y al compromiso con la justicia social. Dichas investigaciones se han desarrollado, sobre todo, a partir del análisis de las presencias y las ausencias de colectivos concretos sobre ejes de identidad específicos como el género, la etnia o la edad.

${ }^{1}$ Asociación Universitaria de Profesorado de Didáctica de las Ciencias Sociales. 
Pagès planteaba las ausencias y las presencias, las personas protagonistas e invisibilizadas, en relación con la proyección de la ciudadanía global, entendiendo que:

los seres humanos de cualquier lugar del planeta ríen y lloran de la misma manera, se reproducen y mueren biológicamente igual, se alimentan, se comunican, viven en un lugar, se protegen de la naturaleza e interactúan con ella, se organizan, etc., pero lo hacen de manera distinta generando esto que hemos denominado cultura (Pagès, 2019a, p.6).

El camino hacia esta proyección de una identidad global humana, sin exclusiones ni invisibilidades, pasaría por el abandono de los planteamientos nacionales y eurocéntricos de la enseñanza de las ciencias sociales (Santisteban, Pagès y Bravo, 2018), y por la descolonización, despatriarcalización y des mercantilización de los contenidos (Santos y Aguiló, 2019). Seguramente es un proceso de ida y vuelta, en la que las temáticas no centradas en procesos geopolíticos favorecen el fin de las exclusiones, y el reconocimiento y visibilización de todas las personas y grupos favorece la recentralización de las temáticas, de lo nacional a lo humano.

Por un lado, el abandono la centralidad nacional para tomar un enfoque centrado en las personas y los procesos humanos favorece la erradicación de las exclusiones: ¿a quién se puede excluir, si hablamos del hambre y el comer, del morir o del nacer? Por otro lado, el reconocimiento de grupos históricamente excluidos no se entiende desde su inclusión en unos relatos ya construidos sobre las experiencias de las élites sociopolíticas, sino desde el reconocimiento de sus experiencias, espacios, perspectivas y cosmovisiones. Estos nuevos espacios y perspectivas transforman las temáticas, desbordando la limitación de los contenidos actuales. Introducen lo oculto, la resistencia, la ternura.

Cuando el objeto de las ciencias sociales escolares deja de ser los estados y las manifestaciones culturales y empiezan a ser las personas -todas las personas- y las experiencias humanas, la responsabilidad en la toma de decisiones sobre el currículum aumenta, porque el abanico de posibilidades sobre las que decidir se abre a toda la vida humana. Y es cuando podemos recuperar la función de la educación crítica y transformadora: revertir desigualdades y acoger la existencia desde todas sus diversidades.

\section{Educar para la ciudadanía global}

Para Joan Pagès, los planteamientos acerca de la formación ciudadana están influenciados por el Marxismo y de manera puntual por la Escuela de Frankfurt. Para ésta, el propósito de las ciencias sociales es desvelar y comprender las relaciones sociales en las que están inmersas las personas. A partir de lo anterior, entiende que las personas pueden construir procesos de emancipación personal y social, en los que la educación juega un papel central. En este sentido, identificar las ideologías presentes en los discursos y prácticas sociales es un eje central de la formación ciudadana.

En sus investigaciones y publicaciones, las finalidades de la enseñanza de las ciencias sociales, la historia y la geografía tuvieron un lugar relevante y dentro de éstas la ciudadanía era un concepto central. Lo anterior es resultado de la influencia de la Teoría Crítica (por ejemplo, Freire, Apple o Giroux), que se centra en el desarrollo de capacidades de reflexión crítica sobre la 
realidad social, así como en una educación para la transformación de esta, es decir, en la formación de un ciudadano crítico y orientado a la participación (Pagès, 2005: Pagès y Santisteban 2007, Santisteban y Pagès, 2009).

Esta finalidad ha ocupado un espacio relevante en el debate educativo y social de las últimas décadas, y como resultado de éste se ha reafirmado la necesidad de trabajar con mayor intensidad en su cumplimiento (Pagès y Santisteban, 2007). En este contexto ha existido una profusa producción escrita en relación con los aportes de las ciencias sociales a la formación de ciudadanos. Una muestra de ello es la realización de eventos, publicación de revistas, libros y manuales sobre el tema. En los debates sobre la formación de ciudadanos, Joan Pagès ha sido claro en plantear que éste es un aspecto de la enseñanza de las ciencias sociales, la historia y la geografía $\mathrm{y}$, que ha estado presente de diferentes maneras e intensidades en la enseñanza obligatoria y en las clases de ciencias sociales.

En términos curriculares, Joan Pagès era crítico con la instrumentalización del currículo, porqué pensaba que eliminaba las posibilidades de una educación crítica y el desarrollo del pensamiento crítico que permite formar a las nuevas generaciones para participar en la vida política democrática (Pagès, 2005).

Joan Pagès fue reiterativo en proponer que ante esta situación, la didáctica de las ciencias sociales permite articular las contribuciones de las diferentes disciplinas o ciencias de referencia y lo que acontece en la sociedad en general. La formación del profesorado en educación para la ciudadanía debe centrar su atención en aspectos fundamentales de las ciencias sociales y en las relaciones con la educación en ciudadanía (Pagès y Santisteban, 2007).

En las instituciones educativas y en la educación obligatoria, la educación de ciudadanos debe estar presente el Proyecto Educativo de Centro, en el currículo y en la enseñanza de las ciencias sociales, por lo tanto, supera los límites de cualquier asignatura o disciplina. La formación de ciudadanos no solo es una de las finalidades centrales de la educación obligatoria, es además una necesidad para el mantenimiento de la propia democracia.

Consideraba que para lograr lo anterior "[h]ace falta, sin duda, una formación inicial y continuada que prepare al profesorado del área, pero también al conjunto del profesorado, para actuar de manera militante a favor de la democracia" (Pagès, 1998, p. 16). El interés de la formación del profesorado para enseñar ciencias sociales y, de manera concreta, educación para la ciudadanía, refleja un interés de las tendencias internacionales, como por ejemplo, la planteada por Torney-Purta, cuando afirma que la formación inicial del profesorado en educación para la ciudadanía ocupa un segundo plano en la agenda, porque "while policy-makers and educators have paid increasing attention to the importance of civic education, little is know about how teachers' knowledge and beliefs influence students' development in this area" (Torney-Purta, Richardson y Henry, 2005, p. 32).

Las contribuciones de Joan Pagès a la manera como se entiende la formación ciudadana tienen implicaciones sociales y educativas amplias. Éstas se encuentran presentes a lo largo de su obra. Sus planteamientos sirven de fundamento para comprender los desarrollos actuales y las apuestas de futuro. En este sentido, uno de los intereses centrales de los últimos tiempos ha estado centrado en que la formación de ciudadanos contribuya a la consecución de una mayor justicia 
social, algo que dialoga de manera concreta con los planteamientos de la educación enmarcados en la Teoría Crítica y con la Pedagogía Crítica.

Algunas de sus últimas preocupaciones y publicaciones de Joan Pagès se centraron en lo relativo a la educación para la ciudadanía global, porqué consideraba que los problemas sociales y educativos tienen esta connotación, no se pueden circunscribir a fronteras nacionales. Eso es consecuencia de los procesos de globalización económica y tecnológica crecientes (Pagès, 2019, Pagès, García y Gutiérrez, 2019; González, Jara, Pinochet, da Silva y Pagès, 2020) comprendidos desde la perspectiva crítica, que fue su marco de referencia permanente. Sin este, no se puede pensar la educación para la ciudadanía, la enseñanza de las ciencias sociales y la formación del profesorado.

\section{Formar el pensamiento social crítico}

Afirmaba Joan Pagès que lo más importante es enseñar a pensar, que este es el gran propósito de la educación y, también, de la enseñanza de las ciencias sociales, de la geografía y de la historia. Pensar en nuestro espacio, situarnos en un lugar del mundo, situarnos en nuestro tiempo, localizarnos en una comunidad local y en el conjunto de la humanidad y de la vida. Pensar en la sociedad y en nosotros mismos, en el presente, en el pasado y en el futuro. Pensar en aquellos problemas sociales con los que nos levantamos cada día, valorar las informaciones sobre dichos problemas y pensar soluciones. Aprender a pensar de forma crítica sobre la realidad que nos rodea y ser conscientes de los cambios sociales que se están produciendo (Pagès, 1997a).

En uno de sus primeros trabajos, junto a Pilar Benejam (Benejam y Pagès, 1988), establecían qué significaba formar el pensamiento social. Un trabajo que tuvo una gran influencia en estudios e investigaciones posteriores. En este trabajo consideraban que el pensamiento social debe formarse a partir del trabajo con problemas sociales relevantes, favoreciendo un pensamiento crítico y creativo, para valorar la información sobre los problemas y para tomar decisiones. Afirmaban que el pensamiento social debe orientarse a la acción social responsable y comprometida con la mejora de la sociedad. Hace más de treinta años de esta publicación y sus ideas siguen estando de actualidad, aunque, es evidente, el contexto ha cambiado, en especial por la transformación que ha provocado el mundo digital.

Desde hace décadas, los medios de comunicación de masas conforman uno de los espacios de socialización con una mayor influencia sobre la ciudadanía. La educación formal e informal compite y colabora con este como otro espacio de socialización, aunque seguramente con una fuerza inferior. Esta realidad nos lleva al estudio del impacto de los medios en la cultura, en la configuración de las identidades, en las representaciones sociales y en las imágenes del futuro de las personas desde las ciencias de la educación, en especial, desde la didáctica de las ciencias sociales. Estos estudios tienen el propósito de entender cuáles son los conocimientos, percepciones, actitudes, valores o emociones que tienen los y las estudiantes, de todas las etapas, en relación con su entorno social pasado y presente, así como sus expectativas sobre el futuro.

En democracia, la educación obligatoria cumple con la función de socialización política con más o menos éxito, con el objetivo fundamental de la autopreservación. Sin embargo, los medios de comunicación (públicos y privados) suelen tener una mayor repercusión en esta socialización, 
hecho que puede conllevar riesgos y oportunidades. Las investigaciones de Joan Pagès apuntan que los jóvenes relacionan "la política con los medios de comunicación, atribuyendo a estos la hegemonía de la información y del análisis político" y son los que explican qué ocurre en el mundo (Sant y Pagès, 2013, p. 211).

Joan Pagès argumentaba que los y las estudiantes tienen sentimientos de desafección hacia la política de partidos y hacia los medios de comunicación, e identifican a los medios como meros transmisores de la información. De sus conclusiones destacamos el poder que tienen los relatos hegemónicos transmitidos desde los medios bajo esta apariencia de neutralidad. Otras de las investigaciones de Joan Pagès muestran como los relatos históricos solo se identifican como tales cuando tienen su origen en la escuela, o incluso en la familia, en cambio, cuando se dan en los medios de comunicación pueden pasar desapercibidos (González-Monfort, Santisteban, Oller y Pagès, 2013, p. 248).

Con esta perspectiva, Joan Pagès argumentaba que la educación debe ser capaz de convertirse en un contrapunto, ofreciendo no uno sino múltiples relatos y discursos que permitan a los jóvenes desarrollar su pensamiento crítico y comprender la complejidad de su realidad social y su capacidad para influir en ella. Con este fin, sostenía que literacidad crítica es una perspectiva teórica y práctica poderosa para el desarrollo del pensamiento crítico y la deconstrucción de los relatos sociales, y que se proyecta hacia el compromiso, la acción, y la transformación social (Ortega-Sánchez y Pagès, 2017).

En su investigación, Ortega-Sánchez y Pagès (2017) ponen de manifiesto que los y las estudiantes de formación inicial hacen lecturas simplistas y reproducen discursos acríticos cuando analizan productos culturales controvertidos con una fuerte carga de valores e ideología. Estos relatos obvian hacer un análisis crítico sobre la desigualdad, la diferencia y la alteridad presente en los textos e imágenes ofrecidas. Joan Pagès defendió la importancia de desarrollar el pensamiento crítico desde la formación inicial a través del uso de imágenes para visibilizar aquellas personas y grupos invisibilizados, y para concienciar sobre las desigualdades de clase, raza o género.

El anterior estudio muestra la importancia que Joan Pagès otorgaba a la concienciación en valores democráticos para el desarrollo del pensamiento crítico. No uno centrado en el aprendizaje de habilidades cognitivas y/o mecánicas sino como algo propio de la idiosincrasia de las personas. La literacidad crítica tiene más de 'saber ser' que de 'saber hacer'. Para Joan Pagès, al aprendizaje de los valores democráticos en el desarrollo del pensamiento crítico se une la importancia de las emociones, puesto que estas “juegan un papel determinante en el aprendizaje de los contenidos históricos, sociales y geográficos, y son un elemento fundamental en la toma de decisiones de la ciudadanía" (Castellví, Massip y Pagès, 2019, p. 24-25). Los medios de comunicación han sabido utilizar las emociones para transmitir su mensaje, sin embargo, el conocimiento escolar ha intentado esconderlas bajo la apariencia de una racionalidad pura.

El estudio de Castellví, Massip y Pagès (2019) muestra como la información presente en los medios digitales puede llegar a tensionar nuestros valores y despertar emociones que nos impidan hacer argumentaciones fundamentadas. El papel de la educación no debe ser el de eliminar estas emociones, sino el de integrarlas en los procesos de desarrollo del pensamiento 
crítico de forma que podamos entender nuestra realidad social también desde una vertiente emocional, como una característica inherente al ser humano y que no debemos obviar.

El desarrollo del pensamiento crítico desde la literacidad crítica es una tarea esencial en nuestro mundo: un contexto social inundado por los medios de masas, la información y la infoxicación, los debates estériles, las opiniones infundadas y los discursos del odio en las redes sociales. Las investigaciones de Joan Pagès (Castellví, Ballbé y Pagès, 2020) sugieren que es necesario potenciar su desarrollo en todos los niveles educativos -desde la educación infantil a la formación inicial y continua- con el objetivo de formar a una ciudadanía crítica y comprometida con los valores democráticos, consciente de sus emociones y socialmente responsable. Para Joan Pagès, esta debe ser una de las principales metas de la educación en nuestros tiempos.

\section{Temporalidad, conciencia histórica y futuro}

Los trabajos de los años ochenta sobre el tiempo histórico abrieron toda una serie de posibilidades para la mejora de la enseñanza de la historia, anclada hasta ese momento en una interpretación muy parcial de las teorías de Piaget. En España esos trabajos vinieron de la mano de Joan Pagès, inspirado en los trabajos italianos e ingleses (Pagès, 1988, 1989 y 1999; Pagès y Santisteban, 1999). A partir de ese momento se introduce la temporalidad y los operadores temporales en la enseñanza de la historia, y estos componentes poco a poco se irán introduciendo en el currículo. También aparecen las primeras investigaciones y tesis doctorales sobre el tema, que hacen evolucionar el conocimiento sobre la temporalidad, que la sitúan en el contexto de la formación del pensamiento histórico y la conciencia histórica.

El pensamiento histórico ha sido un concepto desarrollado mucho más por la didáctica de la historia que por la historiografía. La preocupación por este concepto desde la educación histórica tiene mucho sentido, ya que se trata de saber cómo las personas construimos la historia, cómo interpretamos las fuentes, cómo contextualizamos los hechos y procesos históricos, cómo los narramos, y cómo pensamos la temporalidad y el cambio, finalmente, cómo relacionamos pasado, presente y futuro. La formación del pensamiento histórico es un aspecto imprescindible en la educación para una ciudadanía democrática (Pagès, 2009). Al mismo tiempo, la última finalidad de la enseñanza de la historia es desarrollar las competencias ciudadanas (Pagès, 2012).

Por otro lado, el concepto de conciencia histórica es un concepto de la filosofía de la historia, recogido y analizado por la didáctica de la historia. Si el pensamiento histórico define las competencias a desarrollar para hacer historia, la conciencia histórica es su finalidad más importante. El pensamiento histórico se forma a partir de las primeras edades, a través de habilidades temporales, narrativas, empáticas o interpretativas, al mismo tiempo que se desarrolla la conciencia histórica, de gran complejidad, pero objetivo final del proceso de aprender a pensar históricamente (Santisteban, González-Monfort y Pagès, 2010).

En uno de sus últimos trabajos, Joan Pagès afirmaba que quien se preocupa por el pasado es porque también está preocupado por el futuro (Pagès y Santisteban, 2008; Pagès, 2019b). Sus referencias a la necesidad de desarrollar la conciencia histórica se han traducido en tesis doctorales y en investigaciones, sobre cómo relacionar el pasado con el presente y el futuro. La conciencia histórica forma parte de lo que Freire defendió como conciencia ciudadana crítica. 
Además, la preocupación por la conciencia histórica nos lleva a la educación para el futuro, que no puede prescindir del conocimiento del pasado:

Preparar a los y a las jóvenes estudiantes para "su futuro papel de ciudadanos del mundo" ha sido una de las principales finalidades de aquellos y aquellas docentes que han creído que la enseñanza de la historia debía aportar algo más que lo que proponían los currículos y los programas, basados en las historias nacionales y eurocéntricas y en la memorización de hechos y personajes relevantes de su pasado. Ha sido la preocupación fundamental de quienes han apostado, y siguen apostando, por la formación del pensamiento y de la conciencia histórica y por la acción transformadora que sus saberes pueden facilitar a la ciudadanía (Pagès, 2029b, 24).

Los estudios sobre la memoria histórica han servido para reivindicar el pasado ocultado, silenciado, la narración de los perdedores, que vieron calladas sus voces, ante el relato de los vencedores (Pagès y González Amorena, 2009). Para Rüsen (2007), la memoria rescata el pasado y la conciencia histórica parte del pasado para pensar el futuro. La memoria se relaciona con la empatía y con el juicio histórico. La conciencia histórica está más relacionada con las capacidades para la interpretación y la narración histórica (Rüsen 2001, 2007). La memoria es la lección moral pendiente, mientras que la conciencia histórica es el análisis de las posibilidades y la evaluación de los cambios y continuidades (Pagès y Santisteban, 2008).

Para Dewey (1985) el estudio de la historia parte de los problemas del presente, que pueden tener las raíces en el pasado, pero que, en todo caso, nos permite afrontar la realidad y los conflictos actuales que caracterizan las problemáticas de nuestra sociedad y nuestros problemas, y construir un futuro mejor (Pagès, 2008). Así, la historia se convierte en un campo de conocimiento que nos permite tener un proyecto social, la historia es, entonces, un instrumento de transformación social (Fontana, 1982). La historia explica el presente y nos ayuda a imaginar otro futuro. El currículum se nutre, en este caso, de los temas controvertidos de la actualidad y se convierte en un estudio vivo, significativo y funcional (Pagès, 2015; Santisteban y Pagès, 2019a).

\section{La investigación y la formación del profesorado}

Una de las principales tareas del grupo GREDICS, que Joan Pagès creó y coordinó, ha sido realizar estudios que relacionen investigación e innovación, desde una concepción normalizada del campo científico de la enseñanza y el aprendizaje de las ciencias sociales. Una investigación que solucione los problemas de la práctica y que sea percibida por el profesorado como algo pertinente y necesario. Desde esta misma perspectiva, lo que sucede en la universidad cuando se forma al profesorado, también requiere de investigación e innovación, ya que, en general, no sabemos lo que sucede en las aulas universitarias, qué se enseña y cómo. En este sentido, Joan Pagès ha dirigido una gran cantidad de tesis doctorales sobre la formación inicial y, al mismo tiempo, ha realizado estudios que han sido referentes imprescindibles a quien ha querido adentrase en este campo (Pagès, 1997b, 1998, 2000a).

La investigación sobre las representaciones sociales de los y las estudiantes en formación inicial y, al mismo tiempo, la investigación sobre sus perspectivas prácticas (Pagès, 2000b). Es decir, por un lado, qué piensan los y las estudiantes sobre el tiempo histórico, sobre la política y la participación, sobre los problemas sociales, sobre los conceptos o los valores sociales, sobre los 
derechos humanos o la justicia social... Y, por otro lado, cómo piensan o cómo planifican la enseñanza del conocimiento social. Estas dos vertientes conforman dos aspectos esenciales de la didáctica, la reflexión sobre los contenidos y la reflexión sobre el proceso de enseñanza y aprendizaje. Dos aspectos que requieren investigación y que no se pueden separar.

Enseñamos como hemos sido enseñados, no como nos han dicho que hemos de enseñar. Esta máxima debería traducirse en investigaciones que ayuden a mejorar la formación inicial para la enseñanza de las ciencias sociales. En primer lugar, deberían revisarse los contenidos que se imparten en las facultades de ciencias de la educación. Después, sería necesario adecuar la formación inicial al contexto social actual, en dos sentidos. Uno, para incorporar los problemas sociales y de la escuela en los programas. Dos, para comprender que hoy día la memorización de conocimientos no tiene sentido y que hemos de enseñar a seleccionar e interpretar la información, y hemos de enseñar a pensar.

Pero, ¿qué tenemos o qué hemos construido?, ¿existe suficiente investigación para cambiar los programas y las prácticas en la formación inicial? Creemos que sí, ya que, como cualquier campo científico, la didáctica de las ciencias sociales está en constante construcción o reconstrucción. En la actualidad hemos acumulado una gran cantidad de investigaciones, tesis doctorales, publicaciones, propuestas educativas, etc., para poder transformar la formación inicial del profesorado que ha de enseñar ciencias sociales (Pagès y Santisteban, 2014). Así lo pensaba Joan Pagès y así lo defendemos quienes seguimos sus ideas.

En el II Simposio de la AUPDCS celebrado en Córdoba en 1990, se organizó un debate sobre los programas de formación inicial del profesorado para las diferentes etapas educativas. Los debates fueron coordinados por Rosa M. Ávila, Roser Batllori, Javier de Prado y por Joan Pagès. Este encuentro tuvo una gran importancia para las universidades españolas que, a partir de aquel momento, tuvieron un referente común para un área que por entonces contaba con poca investigación y con pocos espacios de encuentro (AUPDCS, 1990). En 2019, en el simposio de Lisboa, realizamos una revisión de aquella propuesta, que recoge la investigación en formación inicial (Santisteban y Pagès, 2019b). La AUPDCS ha hecho una gran labor en este sentido y ha facilitado siempre la reflexión sobre los contenidos del área para la formación inicial.

Las aportaciones de Joan Pagès a la mejora de la formación inicial del profesorado en didáctica de las ciencias sociales son incuestionables. Dirigió 40 tesis doctorales, de las cuales una buena parte fueron sobre la formación inicial del profesorado o sobre su desarrollo profesional. Uno de sus trabajos marcó un antes y un después en la investigación sobre la formación inicial en nuestra área, en concreto la ponencia presentada en el VII Simposio de la AUPDCS en la Universidad de Las Palmas de Gran Canaria (Pagès, 1997b), trabajo donde se revisaban las principales aportaciones de los diferentes países a la formación inicial para enseñar ciencias sociales y donde se marcaban las líneas de futuro de la investigación.

\section{Identidad, preguntas y comunidad científica}

Cuando tratamos sobre la identidad profesional en la didáctica de las ciencias sociales, nos estamos refiriendo a quien reconoce y se identifica con una comunidad científica, que utiliza una serie de conceptos, métodos y procesos de investigación-innovación, que pueden reconocerse 
como propios. Pero tal vez lo que más nos caracteriza y nos diferencia del resto de comunidades científicas, son las preguntas que nos formulamos en la investigación. A estas preguntas contribuyó como nadie el maestro Joan Pagès, entre otras muchas: ¿qué aporta la enseñanza de las ciencias sociales, la geografía y la historia a la educación cívica?, ¿cómo se enseña y se aprende el espacio geográfico y el tiempo histórico des de la educación infantil hasta cualquier edad?, ¿cómo se enseña y cómo se aprende a participar en democracia?,¿cómo se enseña a pensar, cómo se forma el pensamiento social, histórico, geográfico o el pensamiento crítico?, ¿cómo investigamos e innovamos en educación política, económica, jurídica?, ¿cómo educar para la justicia social desde la enseñanza de la geografía y la historia?, ¿qué aporta la enseñanza de las ciencias sociales a una ciudadanía crítica?, ¿qué aporta a la educación para la justicia social?

Buscando esa identidad, contactó con colectivos internacionales italianos, franceses, ingleses, finlandeses, portugueses, norteamericanos o latinoamericanos, con los cuales hemos compartido esa identidad y las inquietudes por cómo mejorar los estudios sociales. Gracias a esos contactos pudimos incorporar a la didáctica de las ciencias sociales algunos conceptos que se debatían en cada momento en otros países, como los problemas sociales relevantes en Estados Unidos, la enseñanza del tiempo histórico o el laboratorio de historia o geografía en Italia, las habilidades históricas o geográficas en Inglaterra, las representaciones sociales o las cuestiones socialmente vivas en Francia, las situaciones-problema en geografía o en historia en Canadá, o la educación para la justicia social en Latinoamérica, por citar algunos ejemplos. Y pudimos contactar con autores como Ivo Mattozi, Nicole Tutiaux-Guillon, François Audigier, Arja Virta, Peter Seixas, Ian Davies, Ronald W. Evans, Linda Levstik, Keith Barton, E. Wayne Ross, por citar unos pocos.

Con América Latina Joan Pagès mantuvo una relación especial, se sentía como en casa en muchos países donde, después de su partida, se le han realizado múltiples homenajes, algunos de los cuales están todavía pendientes. En América Latina se le reconoce como un autor imprescindible en la configuración del área en sus universidades. Joan Pagès ayudó en muchos países latinoamericanos a construir másteres y doctorados, visitó infinidad de universidades, colegios o cualquier institución que se lo pidiera, impartió conferencias, clases en postgrados o a estudiantes universitarios, a profesores y profesoras de universidad, ayudó a confeccionar programas y proyectos con una gran generosidad, y es recordado por estos motivos con un gran cariño en todas partes y por todo el mundo.

Joan Pagès recibió tres grandes homenajes en vida. En febrero de 2018 se le rindió homenaje en la UAB, en el marco de las XV Jornadas Internacionales de Investigación en Didáctica de las Ciencias Sociales (Ballbé, González-Monfort y Santisteban, 2019). En abril de 2018 se le realizó un gran homenaje en la Universidad Tecnológica de Pereira, organizado por esta universidad y por las de Antioquia y Caldas, además de la Red Colombiana de Grupos de Investigación en Didáctica de las Ciencias Sociales. Y en octubre del mismo año, en el marco del IV Encuentro Iberoamericano de Investigación en Didáctica de las Ciencias Sociales, celebrado en San Carlos de Bariloche, se le sorprendió con un libro que recogía aportaciones de 33 autores de Iberoamérica y prologado por Nicolás de Alba, Ernesto Gómez, y Sebastián Plá (Jara y Santisteban, 
2018). Y en 2021, editado en Brasil, aparecerá un libro homenaje, escrito por 21 profesores y profesoras de España y Brasil (Lima y Santisteban, en prensa).

\section{Para no acabar, sino volver a comenzar, gracias}

Gracias a todas las personas que han colaborado en este número con sus excelentes aportaciones, mostrando las enseñanzas de Joan Pagès, difundiéndolas y ampliándolas, reflexionando en las temáticas que le preocuparon y que siguen abiertas. Gracias por recoger el relevo, por no dejar que se olvide todo aquello por lo que vale la pena luchar, desde la enseñanza de las ciencias sociales, la libertad, la igualdad, la solidaridad, la justicia, la visibilización de todas las personas, grupos e identidades, la humanidad, la diversidad, la ciudadanía crítica, la democracia, la esperanza, el futuro...

Gracias por continuar el debate, por el esfuerzo de buscar respuestas y de aportar nuevas preguntas, como el mejor homenaje que le podemos hacer al maestro.

\section{Referencias bibliográficas}

AUPDCS (1990). Boletín de Didáctica de las Ciencias Sociales, 2. Córdoba: Asociación Universitaria de profesorado de Didáctica de las Ciencias Sociales. Disponible en: http://didacticaciencias-sociales.org/wp-content/uploads/2019/01/2-1990.pdf.

Ballbé, M; González-Monfort, N. \& Santisteban, A. (eds.). Quin professorat, quina ciutadania, quin futur? Els reptes de l'ensenyament de les ciències socials, la geografia i la historia. Barcelona: GREDICS/UAB.

Benejam, P. y Pagès, J. (1988). Ciclo Superior. En M. Moreno y G. Sastre (dir.). Enciclopedia Práctica de Pedagogía. Técnicas Pedagógicas II. Vol. IV. (pp. 282-306). Barcelona: Planeta.

Castellví, J., Ballbé, M., y Pagès, J. (2020). La literacidad crítica digital en el grado de educación primaria: un estudio con maestras y maestros en formación. [Manuscrito presentado para publicación]. https://doi.org/10.13140/RG.2.2.25522.84161

Castellví, J., Massip, M., y Pagès, J. (2019). Emociones y pensamiento crítico en la era digital: un estudio con alumnado de formación inicial. Reidics. Revista de Investigación en Didáctica de las Ciencias Sociales, 23-41. https://doi.org/10.17398/2531-0968.05.23

Dewey, J. (1985). La significació de la geografía i de la història. En J. Dewey, Democràcia i escola (pp. 148-149). Vic: Eumo.

Fontana, J. (1982). Historia: análisis del pasado y proyecto social. Barcelona: Crítica.

Garcés, M. (2020). Escola d'aprenents. Barcelona: Galàxia Gutenberg.

González, G., Jara, M., Pinochet, S., da Silva, L. \& Pagès, J. (2020). Teacher Education and Global Citizenship Education in Latin America: The cases of Argentina, Brazil, Chile and Colombia. In Schugurensky, D \& Wolhuter, C. Global Citizenship Education in Teacher Education: Theoretical and Practical Issues. (pp. 250-263) Routledge: New York

González-Monfort, N., Santisteban, A., Oller, M., y Pagès, J. (2013). La influencia de los medios de comunicación en la construcción del conocimiento Histórico de los alumnos de primaria y secundaria. En J.J. Díaz, A. Santisteban, y A. Cascajero (eds.). Medios de comunicación y 
pensamiento crítico. Nuevas formas de interacción social (pp. 241-251). Alcalá de Henares: Servicio de publicaciones de la Universidad de Alcalá / AUPDCS.

Jara, M.A. y Santisteban, A. (2018a). Los retos de futuro en la enseñanza de las ciencias sociales, la historia y la geografía. En M.A. Jara y A. Santisteban (Coords). Contribuciones de Joan Pagès al desarrollo de la didáctica de les ciencias sociales, la historia y la geografía en Iberoamérica. (pp.273-284). Universidad Nacional del Comanche y Universitat Autònoma de Barcelona.

Jara, M.A. y Santisteban, A. (2018b). (Coords). Contribuciones de Joan Pagès al desarrollo de la didáctica de les ciencias sociales, la historia y la geografía en Iberoamérica. Neuquén: Universidad Nacional del Comanche y Universitat Autònoma de Barcelona.

Lima, C.A. y Santisteban, A. (orgs.) (en prensa). O ensino de história no Brasil e Espanha / La enseñanza de la historia en Brasil y España. Porto Alegre: Editora Fi/ Universidade Estadual de Feira de Santana.

Massip Sabater, M., Castellví Mata, J.y Pagès Blanch, J. (2020). La historia de las personas: reflexiones desde la historiografía y de la didáctica de las ciencias sociales durante los últimos 25 años. Panta Rei: revista digital de Historia y didáctica de la Historia, 167-196. https://doi.org/10.6018/pantarei.445831

Ortega-Sánchez, D., y Pagès, J. (2017). Literacidad crítica, invisibilidad social y género en la formación del profesorado de Educación Primaria. Reidics. Revista de Investigación en Didáctica de las Ciencias Sociales, 102-117. https://doi.org/10.17398/2531-0968.01.102

Pagès, J. (1988). Situar-se en el temps, situar-se en la història. Guix. Elements d'Acció Educativa, 124, 11-16.

Pagès, J. (1989). Aproximación a un currículum sobre el tiempo histórico. En J. Rodríguez Frutos (ed.). Enseñar historia. Nuevas propuestas (pp. 107-138). Barcelona: Laia/Cuadernos de Pedagogía.

Pagès, J. (1997a). La formación del pensamiento social. En Benejam, P. y Pagès, J. (eds.). Enseñar y aprender ciencias sociales, geografía e historia (pp. 151-168). Barcelona: Horsori.

Pagès, J. (1997b). La investigación sobre la formación inicial del profesorado para enseñar ciencias sociales. AUPDCS (ed.). La formación del profesorado y la Didáctica de las Ciencias Sociales (pp. 49-86). Sevilla: Díada.

Pagès, J. (1998). ¿Qué maestro necesitamos para hacer frente a los retos del futuro? En M.V. Sotomayor; A. Rodríguez Marcos; E. Sanz (coord.). La formación de los maestros en los países de la Unión Europea (pp. 325-335). Madrid. Narcea.

Pagès, J. (1999). El tiempo histórico: ¿Qué sabemos sobre su enseñanza y su aprendizaje? Análisis y valoración de los resultados de algunas investigaciones. AA.VV. Aspectos didácticos de Ciencias Sociales 13 (pp. 241-278). Zaragoza. ICE-Universidad de Zaragoza.

Pagès, J. (2000). La didáctica de las ciencias sociales en la formación inicial del profesorado. Iber. Didáctica de las Ciencias Sociales, Geografía e Historia, 24, 33-44.

Pagès, J. (2000b). El currículo de Didáctica de las Ciencias Sociales en la formación inicial del profesorado: investigaciones sobre la enseñanza y el aprendizaje de la DCS. J. Pagès; J. Estepa y G. Travé (eds.). Modelos, contenidos y experiencias en la formación del profesorado 
de Ciencias Sociales (pp. 41-58). Huelva: AUPDCS/Publicaciones de la Universidad de Huelva.

Pagès, J. (2005). Educación cívica, formación política y enseñanza de las ciencias sociales, de la geografía y de la historia. Íber. Didáctica de las Ciencias Sociales, Geografía e Historia, 44, 4551.

Pagès, J. (2008). Ensenyar l'actualitat, per a què? Perspectiva Escolar, 322, 2-11.

Pagès, J. (2009). El desarrollo del pensamiento histórico como requisito para la formación democrática de la ciudadanía. Reseñas de Enseñanza de la Historia, 7, 69-91.

Pagès, J. (2012). Las competencias ciudadanas, una finalidad de la enseñanza de la historia. In S. Plá; X. Rodríguez Ledesma y V. Gómez Gerardo (coord.). Miradas diversas a la enseñanza de la historia (pp. 19-66). México: Universidad Pedagógica Nacional.

Pagès, J. (2015). La educación política y la enseñanza de la actualidad en una sociedad democrática. Educaçâo em Foco, vol. 19 (3), 17-37.

Pagès, J. (2019a). Ciudadanía global y enseñanza de las Ciencias Sociales. Retos y posibilidades para el futuro. REIDICS: Revista de Investigación en Didáctica de las Ciencias Sociales, 5, 5-22.

Pagès, J. (2019b). Enseñar historia, educar la temporalidad, formar para el futuro. El Futuro del Pasado, 10, 19-56. http://dx.doi.org/10.14516/fdp.2019.010.001.001

Pagès, J. y González Amorena, M. P. (coords.) (2009). Història, memòria i ensenyament de la història: perspectivas europees i llatinoamericanes. Barcelona: Servei de Publicacions de la Universitat Autònoma de Barcelona.

Pagès, J. y Santisteban, A. (1999). La enseñanza del tiempo histórico: una propuesta para superar viejos problemas. AUPDCS (ed.). Un currículum de Ciencias Sociales para el siglo XXI (pp. 187207). Diada: Universidad de La Rioja.

Pagès, J. y Santisteban, A. (2007). La educación para la ciudadanía hoy. Educación para la ciudadanía. Madrid: Wolters Kluwer. Recuperado el 30 de septiembre de: 2009. http://www.guiasensenanzasmedias.es/temaESO.asp?tema=4\&materia=ciuda \&dir=\&nod $0=2$

Pagès, J. y Santisteban, A. (2014). Una mirada desde el pasado al futuro en la didàctica de las Ciencias Sociales, en J. Pagès y A. Santisteban, (eds.). Una mirada al pasado y un proyecto de futuro. Investigación e innovación en didáctica de las ciencias sociales (pp. 17-39). Barcelona: AUPDCS/Servei de Publicacions de la UAB, vol.1.

Pagès, J., García, C. \& Gutiérrez, M (2019). Education for Citizenship in the Latin American Context. In J. Pineda-Alfonso, N. De Alba-Fernández y E. Navarro-Medina (eds.). Handbook of Research on Education for Participative Citizenship and Global Prosperity (pp. 195-218). Pennsylvania: IGI Global.

Rüsen, J. (2001). What is Historical Consciousness? - A Theoretical Approach to Empirical Evidence, Paper presented at Canadian Historical Consciousness in an International Context: Theoretical Frameworks, University of British Columbia, Vancouver, BC.

Rüsen, J. (2007). Memory, history and the quest for the future. Cajani, L. (ed.). History Teaching, Identities and Citizenship (pp. 13-34). European Issues in Children's Identity and Citizenship, 7, CiCe. London: Trentham Books. 
Sant, E., y Pagès, J. (2013). La influencia de los medios de comunicación en la socialización política de los jóvenes de 12-16 años en un contexto de crisis económica. En J.J. Díaz, A. Santisteban, y A. Cascajero (eds.). Medios de comunicación y pensamiento crítico. Nuevas formas de interacción social (pp. 207-217). Alcalá de Henares: Servicio de publicaciones de la Universidad de Alcalá / AUPDCS.

Santisteban, A. \& Pagès, J. (2019). Els temes controvertits a l'escola. Perspectiva Escolar, 408, 7-12. https://www.rosasensat.org/revista/temes-controvertits-num-408/els-temescontrovertits-a-lescola/

Santisteban, A. y Pagès, J. (2019). Una nueva lectura de los programas de estudios para la formación inicial del profesorado de didáctica de las ciencias sociales: mirando el presente y el futuro. M.J. Hortas, y A.Dias (eds.). Ensinar e aprender em Didática das Ciências Sociais. A formação de professores numa perspetiva sociocrítica (pp.128-138). Lisboa: AUPDCS / Politécnico de Lisboa / Escola Superior de Educação.

Santisteban, A., Pagès, J. y Bravo, L. (2018). History Education and Global Citizenship Education. En I.Davies, L. HO, D. Kiwan, C. Peck, A. Peterson, E. Sant y Y. Waghid. (Eds.). The Palgrave Handbook of Global Citizenship and Education (pp.457-472). London: Palgrave MacMillan.

Santisteban, A., y Pagès, J. (2009). Una propuesta conceptual para la investigación en educación para la ciudadanía. Revista Educación y Pedagogía, 21, 53, 15-31.

Santos, B. de S. (2019). El fin del imperio cognitivo. La afirmación de las epistemologías del sur. Madrid: Trotta.

Santos, B. de S., y Aguiló, A. (2019). Aprendizajes globales. Descolonizar, desmercantilizar y despatriarcalizar desde las epistemologías del Sur. Barcelona: Icaria.

Seixas, P. (2012). Historical agency as a problem for researchers in history education. Antíteses, 5 (10), 537-353.

Torney-Purta, J., Richardson, W., y Henry, C. (2005). Teachers' Educational Experience And Confidence In Relation To Students' Civic Knowledge Across Countries. International Journal of Citizenship and Teacher Education, (1), 32-57.

Villalón, G. y Pagès, J. (2013). ¿Quién protagoniza y cómo la historia escolar? La enseñanza de la historia de los otros y de las otras en los textos de estudio de Historia de Chile de educación primaria. Clío \& Asociados. La Historia Enseñada, 17, 119-136. 